\title{
Les conséquences de la guerre contre le terrorisme sur le monde des ONG
}

Le cas du réseau de partenaires de Cordaid à travers le monde

The effects of the war on terrorism on the world of NGOs: the case of the Cordaid worldwide partner network

François Lenfant, Lia van Broekhoven et Frank van Lierde

\section{(2) OpenEdition}

Journals

Édition électronique

URL : http://journals.openedition.org/conflits/17779

DOI : $10.4000 /$ conflits. 17779

ISSN : 1777-5345

Éditeur :

CCLS - Centre d'études sur les conflits lilberté et sécurité, L'Harmattan

Édition imprimée

Date de publication : 15 décembre 2009

Pagination : 67-86

ISBN : 978-2-296-11655-9

ISSN : 1157-996X

Référence électronique

François Lenfant, Lia van Broekhoven et Frank van Lierde, «Les conséquences de la guerre contre le terrorisme sur le monde des ONG », Cultures \& Conflits [En ligne], 76 | hiver 2009, mis en ligne le 03 mai 2011, consulté le 30 mars 2021. URL : http://journals.openedition.org/conflits/17779 ; DOI : https:// doi.org/10.4000/conflits. 17779 


\section{Les conséquences de la guerre contre le terrorisme sur le monde des ONG}

Le cas du réseau de partenaires de Cordaid à travers le monde François LENFANT, Lia van BROEKHOVEN, Frank van LIERDE

François Lenfant est doctorant à l'Université d'Amsterdam. Sa thèse porte sur la contribution des firmes multinationales aux processus de paix dans trois pays d'Afrique centrale depuis les années 1990 jusqu'à nos jours. Il est coordinateur des politiques de financement et des politiques de partenariat à Cordaid.

Lia van Broekhoven est conseiller politique à Cordaid, directrice de l'unité Mesures anti-terroristes et responsable du projet cofinancé par Cordaid relatif à l'impact des mesures anti-terroristes sur les partenaires de Cordaid.

Frank van Lierde est conseiller à la communication au sein de Cordaid.

T'essor du rôle des ONG en tant qu'acteurs exerçant une influence croisLsante dans les relations internationales a fait l'objet de nombreuses recherches ces dix dernières années ${ }^{1}$. Pour beaucoup de chercheurs, ce phénomène résulte des défaillances aussi bien du secteur public (gouvernement) que privé (marché). Ces défaillances sont d'autant plus manifestes dans les nombreuses situations de crises humanitaires où les ONG, aussi bien locales qu'internationales, se sont vu attribuer de plus en plus de responsabilités. Dans de telles situations, le rôle qui leur est désormais dévolu inclut non seulement la protection des personnes et la prévention d'abus à leur encontre, mais aussi la mise en place de mécanismes d'alerte et le plaidoyer pour les droits de la personne, la socialisation et le renforcement de la cohésion sociale, la médiation, les activités de consolidation de la paix, etc. ${ }^{2}$ L'interpénétration des domaines de l'humanitaire, du développement et de la transformation des

1. Voir notamment Hernandez V. (dir.), L'action collective à l'épreuve de la globalisation, Paris, L'Harmattan, 2007 ; Troubé C., L'humanitaire en turbulences, Paris, Autrement, 2006 ; Ghandour A.-R., Jihad humanitaire. Enquête sur les ONG islamiques, Paris, Flammarion, 2002 ; Siméant J. et Dauvin P., ONG et humanitaire, Paris, L'Harmattan, 2004 ; Kaldor M., Anheier H., Glasius M., "Global Civil Society in an Era of Regressive Globalisation" in Kaldor M., Anheier H., Glasius M. (eds.), The Global Civil Society Yearbook 2003, Oxford, Oxford University Press, 2003. 
conflits a été finement analysée par Mary Anderson ${ }^{3}$. À ses yeux, l'aide humanitaire ne doit pas seulement être fournie de façon responsable afin de minimiser son impact sur le conflit, mais elle doit aussi réduire les tensions entre combattants. Cette extension des activités des ONG dans des domaines que l'on peut qualifier de politiques rend encore plus complexes et ambiguës les relations déjà tendues que les ONG, aussi bien nationales qu'internationales, entretiennent avec les gouvernements ${ }^{4}$. En effet, ces derniers ont tendance à appliquer une définition restrictive de l'humanitaire, ce qui leur permet de mettre en question la légitimité des ONG à intervenir hors de ce cadre prédéfini ${ }^{5}$. Suite aux nombreuses crises humanitaires des années 1990 qui ont mis en exergue les déficiences inhérentes à une stricte application du droit international humanitaire dans la protection des populations ${ }^{6}$, les ONG ont reconstruit leur légitimité en s'appuyant sur un système de valeurs morales inspirées de la déclaration universelle des droits de l’homme. Néanmoins, que leur légitimité soit fondée sur un socle normatif légal ou moral, les ONG travaillant dans des situations de conflit sont confrontées à de nombreux dilemmes, en particulier celui qui renvoie à leur positionnement vis-à-vis des gouvernements. Cette tension qui caractérise les relations entre gouvernements et ONG en ce qui concerne la définition et le champ d'intervention de l'humanitaire a été exacerbée par le contexte de la «guerre contre le terrorisme ».

Décrétée et mise en œuvre par l'administration des Etats-Unis sous la présidence de George W. Bush en réaction aux attaques du 11 septembre 2001, la guerre contre le terrorisme met en évidence la difficulté d'établir un juste équi-

2. Cf. Mari Fitzduff, "Civil Societies and Peacebuilding - the new Fifth Estate", paper presented for a seminar on Civil Society - UN interaction for Conflict prevention, 2004, et "The World Bank Civil Society and Peacebuilding”, Washington DC, 2006.

3. Anderson M., Do No Harm: How Aid Can Support Peace or War, Londres, Lynne Rienner, 1999.

4. Sur ce phénomène, voir Lindenberg M., Dobel P., "The Challenges of globalization for northern international relief and development NGOs", Nonprofit and Voluntary Sector Quarterly, vol. 28, n 4, 1999. Il est également intéressant de souligner que les ONG sont généralement définies par ce qu'elles ne sont pas, plutôt que par ce qu'elles sont (organisation non gouvernementale, ou organisation à but non lucratif). Les classifications développées jusqu'à présent rendent compte le plus souvent des éléments suivants : leur origine (national/international), leur domaine d'intervention (développement / humanitaire); leur stratégie d'intervention (plaidoyer, prestation de services sociaux, renforcement des capacités) ; leurs bénéficiaires, ou leur finalité. A ce sujet, $c f$. Vakil A., "Confronting the Classification Problem: Toward a Taxonomy of NGOs", World Development, vol. 25, $\mathrm{n}^{\circ} 12$.

5. Voir sur ce point les articles suivants publiés dans la revue Cultures E Conflits (hiver 2005): Eberwein W.-D., «Introduction : comprendre la complexité d'un champ d'action politique » et «Le paradoxe humanitaire : normes et pratiques » (sur la difficulté d'intégrer une version «politique » de l'humanitaire dans un cadre normatif réducteur tel que le droit humanitaire international) ; Heintze H.-J., « Recoupement de la protection des droits de l'Homme et du droit international humanitaire (DIH) dans les situations de crise et de conflit » (sur le chevauchement entre le droit humanitaire international et la protection des droits de l'homme). Il convient de rappeler ici que les ONG locales, quel que soit leur domaine d'intervention, tirent leur légitimité de la liberté d'association, lorsque celle-ci est reconnue et appliquée par les gouvernements en question.

6. Voir notamment Uvin P., L'aide complice? Coopération internationale et violence an Rwanda, Paris, L'Harmattan, 1999. 
libre entre la responsabilité fondamentale conférée aux Etats de protéger leurs citoyens et la nécessité de respecter les droits fondamentaux et les libertés des individus. Les dérives liées à la conduite de cette guerre contre le terrorisme suscitent de plus en plus l'intérêt de travaux universitaires qui permettent d'en avoir une vision plus détaillée. Certains se focalisent sur l'essor de pratiques susceptibles de s'avérer liberticides ou d'attenter à la vie privée : par exemple l'essor du recours aux procédures biométriques d'identification et à diverses technologies de fichage, de traçabilité ou de profilage des personnes 7. D'autres ont pu apporter de précieux éclairages sur des excès de nature différente résultant notamment des procédures judiciaires d'exception instituées dans le cadre du camp de Guantánamo, de la mobilisation de techniques d'entretien assimilables à la torture ou encore de la banalisation des interventions militaires dans des pays comme l'Afghanistan et l'Irak ${ }^{8}$. Il est un autre domaine où l'impact des mesures antiterroristes est loin d'être négligeable et qui n'a pourtant que très marginalement retenu l'attention des chercheurs : celui des ONG œuvrant dans l'humanitaire, le développement, la paix et la réconciliation tant dans les pays occidentaux que dans ceux en voie de développement. Cet article tente de combler ce vide en s'intéressant à la manière dont la guerre contre le terrorisme affecte ces organisations et les individus qui y travaillent en réduisant très substantiellement leur marge de manœuvre. Le champ géographique de l'analyse couvre des pays d'Amérique latine (Colombie, Salvador, Guatemala, Nicaragua), d'Afrique (Ouganda, Soudan) d'Asie (Sri Lanka, Malaisie, Inde, Indonésie) et du Moyen-Orient (territoires occupés de la Palestine) dans lesquels Cordaid 9 développe des partenariats avec différentes ONG locales. Malgré des différences notoires en ce qui concerne leur situation géographique, leur niveau de développement, ou leur régime politique, ces pays partagent une caractéristique commune. Ils ont vécu (Salvador, Guatemala, Nicaragua) ou vivent encore des conflits régionaux auxquels est venue se greffer une dimension nouvelle : la guerre contre le terrorisme.

Les sources sur lesquelles repose cette étude ont été obtenues au travers d'échanges avec des représentants d'organisations partenaires de Cordaid dans le cadre de trois ateliers organisés par Cordaid entre janvier et décembre 2008

7. Voir notamment Crettiez X., Piazza P. (dir.), Du papier à la biométrie. Identifier les individus, Paris, Presses de Science Po, 2006 ; Ceyhan A. (dir.), « Identifier et surveiller. Les technologies de sécurité », Cultures E Conflits, $\mathrm{n}^{\circ}$ 64, hiver 2006.

8. Cf. Bigo D., Bonelli L., Deltombe T. (dir.), Au nom du 11 septembre. Les démocraties à l'éprenve de l'antiterrorisme, Paris, La Découverte, 2008. On pourra aussi consulter les rapports publiés par Human Rights Watch ces huit dernières années.

9. Etablie à La Haye, Cordaid (www.cordaid.nl) est une organisation de la société civile à but non lucratif dont la logique d'intervention hors des Pays-Bas est basée sur un partenariat avec des organisations locales de la société civile dans 32 pays d'Afrique, d'Asie et d'Amérique latine. Les interventions de Cordaid par le biais de son réseau de partenaires s'articulent autour de trois axes stratégiques : la lutte directe contre la pauvreté, le renforcement de la société civile et le plaidoyer. Cordaid finance des programmes dans de nombreux secteurs en fonction du contexte et des priorités définies par ses partenaires locaux : santé, bonne gouvernance, paix et reconstruction, humanitaire et développement économique durable. 
sur le thème de l'impact des mesures anti-terroristes sur la société civile ${ }^{10}$. En plus des réflexions émanant de ces ateliers, 35 entretiens individuels ont été conduits auprès de représentants de ces organisations ${ }^{11}$. Complétés ultérieurement par une correspondance régulière via e-mail avec ces acteurs, ces entretiens ont servi à approfondir la réflexion des organisations partenaires de Cordaid sur l'enjeu de la réduction de l'espace des ONG dans l'exercice de leurs fonctions. D'autres sources particulièrement utiles ont été exploitées, notamment les résultats d'un projet de recherche consacré à cette même thématique également coordonné par Cordaid ${ }^{12}$. La réalisation de ce projet a aussi permis à Cordaid de tisser de nouveaux liens avec d'autres organisations directement affectées par la guerre contre le terrorisme qui réduit notamment leur espace politique et opérationnel ${ }^{13}$.

Dans un premier temps, nous évoquons essentiellement la difficulté qu'éprouvent les ONG internationales à maintenir leur autonomie dans un contexte de suspicion inhérent à la guerre décrétée contre le terrorisme. Ensuite, l'accent est davantage mis sur les conséquences des mesures antiterroristes sur les ONG locales partenaires de Cordaid.

\section{La politisation des critères du financement et ses conséquences}

Un grand nombre de mesures législatives adoptées depuis le 11 septembre 2001 ont des incidences significatives sur le monde des ONG et les fondations privées bailleurs de fonds, aussi bien aux Etats-Unis qu'en Europe. Ainsi, aux Etats-Unis, le Patriot Act, les directives volontaires du Trésor (Voluntary Treasury Guidelines) et un nombre important de décrets présidentiels ${ }^{14}$ limi-

10. Ces ateliers se sont tenus à Maastricht (Pays-Bas), à Davao (Philippines) et à Kampala (Ouganda). Ayant réuni plus de 150 représentants de la société civile (des bailleurs de fonds publics et privés, etc.) ainsi que des membres des gouvernements, ils ont servi à éclairer de façon systématique l'impact négatif des mesures antiterroristes sur les organisations de la société civile.

11. Ces 35 entretiens vont être publiés intégralement dans un ouvrage à paraître intitulé Countering the politics of fear.

12. Cf. Cortright D., Lopez G. A., Millar A., Gerber-Stellingwerf L. M., Friend not Foe: Civil Society and the Struggle against Violent Extremism, A report to Cordaid from the Fourth Freedom Forum and Kroc Institute for International Peace Studies at the University of Notre Dame, La Haye, octobre 2008,

http://www.fourthfreedom.org/Applications/cms.php?page_id=280;

et, Cortright D., Lopez G. A., Millar A., Gerber-Stellingwerf L. M., Overdue Process. Protecting Human Rights While Sanctioning Alleged Terrorism, A report to Cordaid from the Fourth Freedom Forum and Kroc Institute for International Peace Studies at the University of Notre Dame, La Haye, avril 2009. Ces deux rapports sont disponibles sur demande auprès des auteurs. Ils ont notamment servi de support à une campagne de sensibilisation des autorités néerlandaises en vue d'assurer une meilleure protection des droits de la personne, et en particulier du droit d'association.

13. Ces organisations sont : Intrac (http://www.intrac.org/), Ford Foundation (www.fordfoundation.org), Urgent Action Fund (www.uaf.org) et Global Fund for Women (www.globalfundforwomen.org).

14. Cf. Wright K., "Redefining development for national security: implications for civil society", Development in Practice, vol. 19, n 6, août 2009. 
tent considérablement l'espace opérationnel de ces organisations et des fondations américaines. Ainsi, la Ford Foundation a-t-elle été obligée de mettre un terme à certains de ses programmes dans des pays musulmans, soupçonnée qu'elle était d'y financer par ce biais des actions considérées comme "antipatriotes ». Certaines organisations bénéficiaires des fonds versés par cette fondation ont d'ailleurs refusé de former un partenariat avec elle, l'accusant d'appliquer à la lettre les dispositions du Patriot Act dans ses contrats de financement ${ }^{15}$. L'une d'entre elles a aussi engagé des poursuites judiciaires à l'encontre du Patriot Act ${ }^{16}$. Le Global Fund for Women 17 a également été contraint de réduire ses partenariats avec des organisations locales dans les pays musulmans accusés d'être des Etats terroristes ou sympathisants de la cause terroriste. Cela a été significativement le cas au Soudan depuis que ce pays a été placé sur la liste américaine des Etats terroristes ${ }^{18}$. Les sanctions financières érigées contre le régime de Khartoum ont eu des répercussions directes sur les ONG qui œuvrent dans de nombreux domaines (humanitaire, promotion de la paix et de la démocratie, etc.) puisque, excepté Care, l'ensemble des ONG des Etats-Unis a quitté ce pays.

15. La Ford Foundation a été créée en 1936 avec pour but la promotion du bien être mondial (www.fordfound.org). En octobre 2004, l'ACLU (American Civil Liberty Union) a refusé de signer un contrat de financement avec cette fondation (contrat d'une valeur de 1,5 million USD) au motif qu'une des clauses de ce contrat (inspirée du Patriot Act) stipulait que les fonds octroyés par la Fondation ne pouvait en aucun cas être utilisés pour des activités « soutenant le terrorisme ou d'autres activités jugées inacceptables ». Selon l'ACLU, cette formulation était trop ambiguë et limitait, de manière intolérable, l'exercice de certaines de ses activités.Voir notamment Strom S., "ACLU Rejects Foundation Grants over Terror Language", New York Times, 19 octobre 2004. Dans le cadre du projet de recherche mené par Cordaid, des représentants de la Ford Foundation ont d'ailleurs admis ne pas avoir d'autre possibilité que d'appliquer les recommandations du Patriot Act, tout en œuvrant auprès des autorités pour que son contenu ne préjudicie pas trop aux actions conduites par certaines organisations de la société civile.

16. Ces poursuites judiciaires ont été engagées par l'ACLU en 2003. En 2006, l'ACLU a retiré sa plainte après que quelques améliorations furent apportées à la législation. Ces améliorations concernaient essentiellement les restrictions imposées au FBI dans les possibilités qui lui étaient accordées de se procurer des informations auprès d'organisations soupçonnées d'être impliquées dans le terrorisme. $C f$. http://www.aclu.org/affiliates/michigan.html

17. Le Global Fund for Women (GFW) est une organisation de promotion des droits de la femme (www.globalfundforwomen.org). Dans le cadre du projet de recherche, un entretien a été réalisé avec Dale Needles, directeur des opérations du GFW, à New York en juillet 2007. Durant cet entretien, il a confié que, à la suite de l'adoption du Patriot Act, le GFW avait été contraint de conduire une politique de financement « prudente ».

18. En août 1993, le département d'Etat des Etats-Unis a accusé le Soudan de soutenir des activités terroristes parce qu'il autorisait sur son territoire une libre circulation de terroristes locaux et internationaux, comme Oussama ben Laden. Ces dernières années, le gouvernement soudanais a affiché son intention de combattre le terrorisme. En réaction, le Conseil de sécurité des Nations unies a levé les sanctions contre Khartoum en 2001. En 2007, les autorités américaines ont déclaré que le Soudan était devenu "un allié important dans la guerre contre le terrorisme». Néanmoins, le Soudan reste sur la liste américaine des Etats terroristes établie par les Etats Unis en raison des liens du gouvernement soudanais avec le Hamas. Cf. Notamment Council on Foreign Relations: http://www.cfr.org/publication/9367/state_sponsors.html. L'ambiguïté des relations entre les autorités soudanaises et les États-Unis, et celle du rôle joué par l'État soudanais dans la lutte contre le terrorisme sont illustrées par les propos de certains partenaires de Cordaid au Soudan (voir infra). 
La charge de la preuve s'est donc en quelque sorte trouvée brusquement inversée. En effet, toute organisation travaillant dans des pays considérés comme « fragiles » ne constituant pas des Etats de droit se doit désormais de prouver qu'elle n'entretient aucune relation avec des terroristes. Une étude menée en 2004 par le Foundation Center ${ }^{19}$ a bien montré ce processus en soulignant combien les ONG éprouvent actuellement des difficultés à financer des activités de nature internationale en vertu de l'avènement de cette nouvelle logique ${ }^{20}$. Dans le même temps, les organisations qui s'investissent dans des programmes de paix et de réconciliation ou de protection des droits humains sont davantage contrôlées en raison de la dimension «politique » de leurs missions. Certains domaines, comme la défense des droits de l'homme ou la lutte contre les inégalités, sont considérés comme très " sensibles ", voire "subversifs ", et connaissent de plus en plus de difficultés de financement par les acteurs gouvernementaux bailleurs de fond. Plus le domaine est perçu comme "délicat ", moins les organisations internationales sont à présent disposées à courir le risque d'y financer des activités : la « politisation » des critères présidant à l'octroi d'aides financières étatiques les incite à s'orienter vers des programmes « sûrs » qui ne seront pas susceptibles d'entacher leur réputation.

Un grand nombre d'ONG internationales subit des pressions gouvernementales. L'obtention d'un contrat de financement pour mener une action au Mali par exemple est subordonnée à l'acceptation de la mise sur pied d'un certain type de programme en Afghanistan ou en Irak (deux pays qui reçoivent une aide publique considérable car rentrant dans le schéma de la guerre contre le terrorisme ${ }^{21}$ ). Cette " politisation » des critères de financement qui conditionne fortement les choix de programmation et l'action du monde de l'humanitaire constitue de facto un moyen de renforcer la politique sécuritaire des gouvernements occidentaux. A cet égard, les chiffres relatifs aux allocations publiques attribuées en faveur de l'aide humanitaire sont éloquents : entre 2001 et 2007, l'aide humanitaire et l'aide au développement pour des pays comme l'Afghanistan et l'Irak ont connu une très nette augmentation, tandis que, dans le même temps, l'aide allouée au Burundi, au Niger ou au Bangladesh augmentait de façon beaucoup moins significative. A titre d'exemple, l'aide publique allouée en Irak a augmenté de $7571 \%$ entre 2001 et 2007, alors que, durant la même période, l'aide au Bangladesh et au Niger n'augmentait respectivement que de $144 \%$ et $213 \%$ respectivement (cf. tableau cidessous) 22 .

19. La Foundation Center est une organisation qui a été instituée en 1956 (www.foundationcenter.org). Elle représente un centre de ressources capitalisant des informations et des outils susceptibles d'aider d'autres fondations dans leur fonctionnement. Elle gère la plus grande base de données concernant les bailleurs de fonds et leurs programmes.

20. Renz L., et Atienza J., International Grantmaking update. A Snapshot of US Foundation Trends, Foundation Center, octobre 2006 :

http://foundationcenter.org/gainknowledge/research/pdf/intl_update_2006.pdf

21. Cf. Fowler A., Aid architecture: Reflections on NGO futures and the emergence of Counter Terrorism, Oxford Intrac, Occasional Paper series $n^{\circ} 45$, janvier 2005 :

http://www.intrac.org/docs/OPS45final.pdf 
Total Bilateral Aid To All Sectors, in USD Millions

\begin{tabular}{|c|c|c|c|c|c|c|c|c|}
\hline & 2001 & 2002 & 2003 & 2004 & 2005 & 2006 & 2007 & $\%$ change \\
\hline & & & & & & & & 2001-2007 \\
\hline Afghanistan & 405 & 1300 & 1591 & 2169 & 2749 & 2999 & 3951 & $976 \%$ \\
\hline Bangladesh & 1046 & 909 & 1394 & 1417 & 1322 & 1222 & 1502 & $144 \%$ \\
\hline Burundi & 139 & 172 & 227 & 359 & 364 & 415 & 466 & $335 \%$ \\
\hline Iraq & 121 & 106 & 2250 & 4647 & 22046 & 8870 & 9176 & $7571 \%$ \\
\hline Niger & 253 & 299 & 461 & 547 & 520 & 514 & 539 & $213 \%$ \\
\hline
\end{tabular}

En ce qui concerne l'allocation de l'aide aux pays où se joue la guerre contre le terrorisme, l'aide n'a pas seulement augmenté en volume. La nature des programmes financés ainsi que les finalités pour lesquelles des fonds ont été alloués dans le cadre de l'aide officielle remettent en cause un des fondements de l'aide au développement : la lutte contre la pauvreté. En Afghanistan par exemple, des «Equipes provinciales de reconstruction » ont été instituées pour rebâtir le pays. Ces équipes sont certes composées de militaires et de membres d'ONG humanitaires, mais les programmes mis en œuvre répondent essentiellement à des exigences militaires ${ }^{23}$. La stratégie mise en avant par les pays occidentaux suit la logique dite des «3D », à savoir : "Défense, Diplomatie, et Développement » ${ }^{24}$.Cette nouvelle logique constitue une ligne directrice à laquelle toutes les ONG humanitaires doivent désormais se plier. Dans la pratique, cela débouche cependant sur une situation dans laquelle l'objectif de défense prend le dessus sur les deux autres : ce qui aboutit à une subordination significative des enjeux humanitaires aux enjeux militaires et sécuritaires ${ }^{25}$. Concrètement, l'approche «3D » encourage un certain niveau de coopération entre les forces militaires et les ONG, ce qui engendre une situation où le domaine de l'humanitaire ne relève plus de la seule responsabilité des ONG. Dans beaucoup de cas, les militaires s'investissent dans des projets humanitaires à haute visibilité afin de conquérir « les cœurs et les esprits » des populations. Outre les critiques portant sur la compétence des militaires pour exécuter de tels projets humanitaires, les relations qui se tissent entre militaires et $\mathrm{ONG}$ dans les pays où se joue la guerre contre le terrorisme posent de nombreux problèmes à ces dernières. Ainsi, une étude consacrée aux relations entre ONG et militaires en Afghanistan a bien montré les risques que cette approche implique pour les ONG. Etre associé aux forces militaires met non seulement en danger la sécurité de leur personnel, mais compromet également leur neutralité, ce qui constitue le socle de leur légitimité ${ }^{26}$. La

22. Comité pour l'aide au développement de l'OCDE, «ODA aid flows, all sectors » (http://stats.oecd.org/qwids).

23. Cf. Cortright D. et al., op. cit., p. 13.

24. Sur la logique 3D, voir notamment Frerks G., Klem B., van Laar S., van Klingeren M., Principles and Pragmatism. Civil - Military Action in Afghanistan and Liberia. Rapport réalisé pour Cordaid, La Haye, 2006.

25. Sur ce point, $c f$. notamment l'article publié par le journal De Volkskrant le 7 septembre 2009 ("Hulpverleners zijn te vaak doelwit » : "Le staff des organisations humanitaires est trop souvent pris pour cible ») faisant état des récriminations formulées par Médecins sans frontières. Hans van de Weerd, directeur de Médecins sans frontières en Hollande y mentionne tout spécialement les dangers auxquels sont confrontés ses membres dans les pays où l'OTAN ou d'autres forces militaires étrangères sont présentes. 
sécurité des équipes des ONG n'est pas uniquement mise en danger par des groupes terroristes. En Afghanistan par exemple, des soldats faisant partie de la force militaire internationale ont envahi un hôpital géré par une organisation partenaire de Cordaid car ils avaient reçu des informations en provenance des services de sécurité leur indiquant qu'un terroriste y était soigné. A la suite de cette opération musclée au cours de laquelle le personnel de l'hôpital ainsi que des patients ont été intimidés, et des dossiers médicaux saisis, certains collaborateurs de l'ONG en question ont démissionné 27. Dans un tel contexte, l'impératif humanitaire est de plus en plus soumis à des pressions politiques et militaires, tandis que l'aide au développement est subordonnée à des enjeux géopolitiques qui sapent la raison d'être du monde humanitaire : fournir une assistance aux personnes en détresse.

\section{Logiques de suspicion/stigmatisation et durcissement des contrôles}

Le FATF (Financial Action Task Force) est un organe intergouvernemental dont l'objectif est le développement et la promotion de politiques nationales et internationales visant à combattre le blanchiment de l'argent et le financement des activités terroristes. Il a émis neuf recommandations aux Etats membres de l'Union Européenne (UE) et aux instances de l'UE relatives au contrôle des flux financiers destinés aux organisations terroristes. L'une d'entre elles, la recommandation VIII, invite les gouvernements et les institutions financières à "s'assurer que les organisations à but non lucratif ne soient pas utilisées à mauvais escient pour financer des activités terroristes 28 ». La plupart de ces recommandations ont été reprises telles quelles dans de nombreuses législations nationales. En outre, le FATF a défini un « code de conduite " pour les ONG dont l'objectif consiste à assurer le contrôle des flux financiers à destination des terroristes. Dans le cadre de ce code de conduite, la preuve de non culpabilité leur incombe. Les partenaires de ces ONG œuvrant dans les pays en voie de développement doivent être passés au crible fin et sont dans l'obligation de signer des documents attestant qu'ils ne constituent pas des organisations terroristes. Outre les conséquences bureaucratiques ${ }^{29}$ et humaines (en particulier l'érosion de la confiance dans les relations entre organisations du Nord et du Sud) induites par l'application de ce code au sein des ONG, la non-adhésion à son contenu a des répercussions non négligeables. Par exemple, dans le cadre de la régulation des ONG aux PaysBas, le CBF 30 (organisme de certification des ONG hollandaises) subit la

26. Cf. Frerks G. et al., op. cit.

27. Entretien avec Paul van der Berg, chargé de programme et coordinateur politique au sein de Cordaid. Entretien réalisé le 9 octobre 2009 à La Haye. L'organisation à laquelle nous faisons référence est une organisation médicale basée dans le sud de l'Afghanistan dont nous ne pouvons révéler ici le nom.

28. www.fatf-gafi.org/dataoecd/43/5/38816530.pdf

29. Lister l'ensemble des organisations partenaires, rassembler toutes sortes de documents les concernant, vérifier ces documents, se prononcer sur la posture à adopter, etc. Sur ce point, cf. Fowler A., op. cit., p. 22. 
pression du gouvernement afin qu'il reprenne à son compte la recommandation VIII du FATF et qu'il y inclue une clause de non financement des organisations à caractère terroriste dans les contrats de partenariat. Dans le contexte hollandais, ne pas obtenir le « label » $\mathrm{CBF}$, qui garantit une légitimité auprès du public, déboucherait sur une irrémédiable et significative baisse des revenus pour les $\mathrm{ONG}^{31}$.

La culture de la peur et de la stigmatisation règne, comme en témoigne une déclaration de Gordon Brown qui a justifié les mesures financières draconiennes prises à l'encontre du secteur des organisations caritatives au Royaume-Uni par la propension qui les caractérise à commettre des abus ${ }^{32}$. Ce type de discours tend à accréditer l'idée selon laquelle ces organisations ne sont pas «professionnelles ", sont faciles à manipuler, et constituent donc une proie facile pour les terroristes. C'est pourquoi, dans cette optique, leur imposer une stricte régulation étatique est présenté comme une absolue nécessité afin d'éviter qu'elles ne basculent dans la «criminalité ». Des études récentes ont pourtant montré que les accusations lancées à l'encontre d'ONG soidisant impliquées dans des activités criminelles étaient infondées ${ }^{33}$. La législation relative à la lutte contre le terrorisme ne se limite plus aux actes terroristes perpétrés, mais vise surtout à tout mettre en œuvre pour les prévenir. Une telle logique proactive est propice au développement d'un climat de méfiance généralisé qui rend beaucoup d'organisations de la société civile des pays du Nord comme du Sud suspectes de soutien aux organisations terroristes.

Le phénomène de «listing/delisting » s'inscrit dans cette même logique d'incrimination tout en permettant de renforcer les contrôles sur le monde de l'humanitaire et du développement. Les procédures officielles de prise de décision relatives à l'inscription d'un individu ou d'une organisation sur une liste terroriste sont loin d'être transparentes. Tout d'abord, il est nécessaire ici de rappeler la distinction qui existe entre le système procédural des Nations unies et celui de l'UE. Les Nations unies connaissent deux systèmes en la matière : le système d'inscription directe géré par les Nations unies et le système d'ins-

30. CBF (Centraal Bureau Fondsenwerving) est un organe indépendant visant à réguler les ONG.

31. Cordaid et d'autres organisations hollandaises ont décidé d'instituer un code de conduite interne dans lequel figure une clause de non financement d'activités terroristes, élaborée selon leur propre définition, et sans intervention gouvernementale.

32. Cf. http://www.publictechnology.net/modules.php? op=modload\&name=News\&file= article\&sid=4449

Prononcé à la Royal Services Institute de Londres, ce discours de Gordon Brown précisait sa vision de la sécurité au Royaume-Uni en abordant diverses thématiques : les cartes d'identité biométriques ou encore le financement des organisations terroristes. Il a alors indiqué être en possession d'informations "selon lesquelles des fonds ont été transférés du Royaume-Uni en Afghanistan pour soutenir des camps de formation, et financer des actes terroristes en Afghanistan et dans d'autres parties du monde, au travers de réseaux sophistiqués auxquels une organisation caritative est liée ».

33. Cf. Sen K., Morris T., Civil Society and the War on Terror, Oxford, Intrac, 2008 ; Quigley N., Pratten B., Security and Civil Society: The Impact of Counter-Terrorism Measures on Civil Society Organizations, Londres, National Council for Voluntary Organizations, 2007. 
cription au niveau national. Dans le premier système, chaque pays membre est habilité à formuler une demande d'inscription auprès du comité de sanction des Nations unies. Dans le second, les Nations unies, via la résolution 1373, adoptée le 28 septembre 2001, encouragent les Etats membres à identifier euxmêmes de manière indépendante les suspects terroristes et à établir nationalement des critères en vue de définir qui doit être considéré comme tel. En ce qui concerne le système d'inscription direct, la résolution 1267 adoptée en 1999 a institué un comité de sanction dont la composition est identique à la celle du Conseil de sécurité ${ }^{34}$. En 2000, la résolution 1333 a demandé au comité de sanction d'actualiser une liste d'individus et d'organisations associés à Oussama ben Laden. Publiée pour la première fois le 8 mars 2001, cette liste contient les noms d'individus et d'entités dont les avoirs doivent être gelés. Par la suite, elle a fait l'objet de modifications (la dernière datant de 2006 à travers la résolution 1735). La résolution 1390 adoptée par les Nations unies le 28 janvier 2002 stipulait que les Etats membres se devaient de prendre des mesures restrictives à l'encontre d'Oussama ben Laden, des membres d'Al Qaeda, des Talibans et de tout individu et groupe associé à Al Qaeda. L'UE a décidé de reprendre telle quelle la liste établie par les Nations unies en application de la résolution 1390 et elle a défini un régime de sanctions applicable aux individus et aux organisations qui y figuraient sans procéder à aucune vérification préalable. Entre temps, la résolution 1373 des Nations unies, adoptée le 28 septembre 2001, a fixé un cadre directeur à partir duquel les Etats membres peuvent définir des régimes de sanction appropriés. Cette résolution précise que les Etats membres s'engagent à prévenir et à supprimer le financement des terroristes, à limiter leur liberté de mouvement et d'action en renforçant leurs contrôles aux frontières, à intensifier les échanges d'information et à contrôler rigoureusement les informations fournies par les demandeurs d'asile avant l'octroi du statut de réfugié. Jusqu'à ce jour, les individus ou organisations qui figurent sur ces listes n'en sont pas avertis. De plus, l'inscription d'une organisation sur une liste n'est pas motivée, et les individus et organisations n'ont aucune possibilité de faire appel. Une fois inscrit sur une liste terroriste, il est aussi pratiquement impossible d'initier une action pour en sortir, comme en témoigne le cas de Yassin Abdullah Kadi, un homme d'affaire saoudien accusé d'entretenir des relations avec Oussama ben Laden ${ }^{35}$.

En réaction à certaines critiques qui ont été avancées pour dénoncer cette situation, les Nations unies ont adopté, en 2006, la résolution 1730. Elle institue au sein du Secrétariat un point de référence qui se voit confier la responsabilité du traitement des demandes formulées par les personnes et entités appa-

34. Les cinq pays membres permanents du Conseil de sécurité (États-Unis, Russie, Chine, France et Royaume-Uni) exercent donc une influence énorme sur la procédure de placement ainsi que sur le régime de sanction.

35. Cf. le Rapport mondial 2009 de Human Rights Watch où est fait référence à un arrêt de la Cour européenne de Justice précisant que le droit d'être entendu et de contester la décision de geler les avoirs de M. Kadi l'emporte sur l'obligation des Etats membres des Nations unies de respecter les mesures prises par le Conseil de sécurité. 
raissant sur la liste afin que soient levées les mesures prises à leur encontre. Ces demandes peuvent être soutenues par les gouvernements des Etats dont sont issues les personnes et entités apparaissant sur une liste. Néanmoins, cette procédure ne constitue en aucun cas un mécanisme transparent de révision. Les acteurs ayant formulé une requête ne sont pas invités à témoigner oralement et la possibilité d'être retiré de la liste est assujettie à l'approbation de l'ensemble des membres du comité de sanction. L'impact du point de référence est donc limité. Suite à la résolution 1822 adoptée en 2008, les Nations unies ont demandé aux Etats membres de réviser la liste, de motiver le maintien du placement de ceux qui y demeureront (les arguments avancés par les Etats sont mis en ligne sur le site Internet des Nations unies) et de justifier tout nouveau placement à partir de juin 2010. Hormis la liste des Nations unies, celle de l'Union européenne, les listes américaine, britannique et indienne, aucune autre liste n'est rendue publique, à l'exception de la liste indienne.

Outre les mesures prises dans le cadre des résolutions adoptées par les Nations unies, l'UE a établi sa propre liste autonome. Sur la base de la résolution 1373 du conseil de sécurité des Nations unies, elle a adopté une position commune en 2001 (2001/931/CFSP) relative aux mesures à instituer pour combattre le terrorisme. Ces mesures sont similaires à celles prises dans le cadre de la résolution 1 373. Le règlement (CE) 2580/2001 dérivant de la position commune de 2001 stipule que l'UE doit établir une liste de personnes et entités suspectes d'activités terroristes sur la base d'informations fournies par les Etats membres. Le Conseil de l'Europe est l'organe habilité à amender ou modifier cette liste sur la base de positions communes : la Commission européenne et le Parlement européen ne jouent aucun rôle dans cette procédure. Dans la pratique, Interpal 36 (une ONG britannique œuvrant dans le domaine de l'humanitaire) a par exemple été indûment accusée de financer des actions terroristes en Palestine. Le simple fait d'être présent et de ne travailler que dans ce territoire a suffi pour que soient formulées à son encontre des accusations de malversation et de terrorisme. L'administration américaine a exercé des pressions sur les gouvernements canadien, australien et britannique afin qu'ils placent cette organisation sur leur propre liste nationale terroriste. Malgré des recherches conduites par l'UK Charity Commission 37 qui ont apporté la preuve de l'innocence d'Interpal ${ }^{38}$, le gouvernement américain refuse toujours aujourd'hui qu'elle soit retirée de la liste terroriste nationale.

36. Interpal est une ONG humanitaire qui soutient les personnes en détresse dans les territoires occupés de la Palestine.

37. L'UK Charity Commission est un organe régulateur des organisations caritatives enregistrées au Royaume-Uni, www.charity-commission.gov.uk

38. Cf. le rapport de la UK Charity Commission en date du 27 février 2009, Cwww.charity-commission.gov.uk/news/printerpal.asp 
La conclusion que l'on peut tirer de cette analyse du phénomène listing/delisting est que, malgré les améliorations apportées récemment, la procédure de placement sur une liste terroriste reste opaque. Il faut également ajouter que ces améliorations, résultat de travaux de plaidoyer effectués par des organisations de la société civile, dont Cordaid ${ }^{39}$, concernent essentiellement le phénomène de listing/delisting européen. Dans les pays en voie de développement où l'Etat de droit et la séparation des pouvoirs ne sont pas institués ou mis en œuvre, les pratiques nationales de listing/delisting répondent à une logique encore plus arbitraire, comme le soulignent les témoignages apportés par les partenaires de Cordaid qui ont pu en être victimes. Ainsi, une personne travaillant à titre individuel avec Cordaid a-t-elle vu son nom circuler sur une liste terroriste aux Philippines, liste qui a été publiée dans la presse locale ${ }^{40}$. Aucune accusation formelle n'avait pourtant été lancée à son encontre. Afin que son nom soit radié de cette liste, cette personne a été contrainte de saisir directement le conseiller du Président en matière de sécurité. Mobiliser constamment ses réseaux et entreprendre des actions de lobbying deviennent des stratégies nécessaires pour les partenaires de Cordaid afin de pouvoir espérer continuer leurs actions. Au Sri Lanka, «les noms et photographies de personnes qui n'ont pourtant aucun lien formel avec le LTTE ${ }^{41}$ [les " tigres Tamoules"] apparaissent pourtant sur des listes gouvernementales simplement parce qu'elles soutiennent les communautés tamoules $42 »$. De manière générale, les participants aux ateliers organisés par Cordaid ont indiqué ne disposer d'aucune information fiable concernant la manière dont sont composées ces listes au niveau national et les éventuelles procédures instituées pouvant servir à contester leur logique. L'existence et la circulation de listes locales et nationales (en parallèle des listes établies au niveau international dont les procédures manquent de transparence) contribuent également à alimenter un climat de peur au sein des ONG. Comme le souligne la responsable de l'organisation soudanaise Gender Centre for Research and Training in Khartoum, les incertitudes qui caractérisent les pratiques du listing et delisting sont d'ailleurs amplifiées par l'absence de mobilisation de critères fiables pour définir ce qu'est un acte terroriste ${ }^{43}$.

\section{Violences et restriction des libertés}

La plupart des partenaires de Cordaid ayant participé aux ateliers ont été victimes de violences physiques et/ou psychologiques. Intimidations constantes, arrestations arbitraires, expulsions momentanées, surveillance

39. Ibid., p. 1 et p. 12.

40. Entretien avec Elias Macarandas, spécialiste de l'étude du Coran. Entretien réalisé à Amsterdam en janvier 2008.

41. Liberation Tigers of Tamil Eelam, un groupe séparatiste au Sri Lanka.

42. Entretien avec Sudarshana Gunawardana, op. cit.

43. Entretien avec Asha El-Karib (fondatrice de Gender Centre for Research and Training in Khartoum et directrice de l'Organisation soudanaise pour la Recherche et le Développement) réalisé à Kampala (Ouganda) en juillet 2008. 
continue constituent des abus auxquels ils sont régulièrement soumis. La plupart de ces abus sont commis par les services de sécurité relevant du gouvernement. Le cas d'une personnalité religieuse (dont l'identité ne peut être mentionnée pour raison de sécurité) travaillant pour une organisation située à Jaffna ${ }^{44}$ est à cet égard révélateur. Le père X, arrêté en 2006 au Sri Lanka pour activité menaçant la sécurité de l'Etat a subi de multiples intimidations et menaces de la part de l'armée ${ }^{45}$ pour avoir organisé une campagne de défense des droits humains. De même, un chercheur colombien a été obligé de fuir son pays après avoir subi des menaces des services de sécurité car ses travaux étaient considérés comme contraires à la ligne politique gouvernementale selon laquelle l'approche militaire contre le narco-terrorisme est couronnée de succès ${ }^{46}$. Dans un autre cas, une tête de mort a été dessinée sur la porte d'une organisation de défense des droits de la femme en Colombie. La dirigeante de cette organisation avait aussi précédemment été menacée par deux personnes lui ayant demandé quel service funéraire elle souhaitait qu'on lui organise ${ }^{47}$. Dans le pire des cas, travailler pour une ONG dans un domaine sensible peut conduire à la mort. L'un des interviewés indique qu'il a recensé une trentaine de personnes tuées dans l'exercice de leur fonction ${ }^{48}$. Les dangers auxquels les travailleurs humanitaires sont exposés ont augmenté ces dernières années. Etre en contact avec des organisations qualifiées de terroristes pour négocier des libérations d'otages - ce qui est au cœur même du travail effectué par certains membres des ONG œuvrant pour la paix - leur vaut d'être sous le feu aussi bien des terroristes que des forces gouvernementales. Ainsi, Maguid Mahurom, représentant d'une ONG défendant les droits de la minorité Moro aux Philippines explique "être constamment pris dans un étau et subir la menace des deux camps 49 ". Ce sentiment d'être victime à la fois de régimes répressifs et de la guerre menée contre le terrorisme dont profitent ces mêmes régimes pour accentuer localement leurs contrôles sur les ONG est partagé par beaucoup de partenaires de Cordaid. Lors des différents ateliers organisés par Cordaid, il est clairement ressorti que l'agenda anti-terroriste international défini sous l'égide de l'administration des Etats-Unis durant la présidence de George W. Bush a grandement servi les agendas sécuritaires nationaux, notamment en légitimant des mesures de restriction du champ d'action des ONG. Dans de nombreux pays, le durcissement des contrôles et le renforce-

44. OMI, Justice, Peace and Integrity of Creation Office.

45. Qui l'ont d'ailleurs forcé à s'exiler aux Philippines en mars 2008.

46. Entretien réalisé en février 2009 avec Ariel Avila, chercheur à l'institut Corporacion Nuevo Arco Iris, une organisation non gouvernementale basée à Bogota partenaire de Cordaid, www.nuevoarcoiris.org.co/sac/

47 . Entretien avec Clara Elena Cardona Tamayo, avocate et dirigeante d'une organisation de protection des droits de la femme Casa de la Mujer, partenaire de Cordaid, La Haye, décembre 2008.

48. Entretien avec Francisco de Roux, prêtre, leader de l'ordre des jésuites en Colombie, Maastricht, janvier 2008.

49. Entretien avec Maguid Mahurom, membre de CBCS (Consortium of Bangsamoro Civil Society), un réseau d'organisations moro luttant pour le respect de leurs droits et la préservation de leur culture. Entretien réalisé à Davao en mai 2008. 
ment des législations sont réalisés en l'absence même d'attaques terroristes. En Malaisie par exemple, Latheefa Koya, avocate et secrétaire générale de l'organisation SUARAM (Suara Rakyat Malaysia, Voice of the Malaysian People) partenaire de Cordaid indique : "Le gouvernement a régulièrement appliqué le Internal Security Act (ISA) 50 contre toute forme de dissidence politique malgré l'absence totale d'incidents terroristes ces dix dernières années. Auparavant, la communauté internationale condamnait les arrestations arbitraires effectuées dans le cadre de l'ISA. Depuis le 11 septembre, ces arrestations suscitent beaucoup moins de critiques. En Malaisie, les mesures anti-terroristes visent à tout contrôler, en particulier les membres des ONG, et contribuent à alimenter un sentiment de révolte parmi la population 51 ».

$\mathrm{Au}$ Salvador, la loi anti-terroriste «Ley Especial contra Actos de Terrorismo » adoptée le 21 septembre 2006 relève de la même logique. Comme le souligne la responsable d'une organisation partenaire de Cordaid travaillant dans ce pays à la promotion des droits de la femme, cette loi est l'expression "d'une politique exclusivement centrée sur la sécurité militaire et non sur la sécurité des citoyens. La liberté citoyenne est violée au nom de la guerre contre le terrorisme 52 ». En Inde, l'Unlawful Activities Prevention Act met l'accent sur l'intention des suspects et leurs opinions. Avec cette nouvelle législation anti-terroriste, comme l'indique Babloo Loitongbam (avocat, dirigeant et fondateur de Human Rights Alert : une organisation de protection des droits humains en Inde), le simple fait de "discuter de l'autonomie politique d'une région dans le nord-est de l'Inde est passible d'une peine de prison, ce qui criminalise des pans entiers de la société civile poursuivant un agenda politique différent de celui de l'Etat 53 ». Les organisations de protection des droits de l'homme telles que Human Rights Watch ou Human Rights Alert ont d'ailleurs abouti à la conclusion que cette législation confère au gouvernement une sorte de carte blanche dans son combat pour la préservation de l'unité et de l'intégrité de l'Etat indien ${ }^{54}$. Au Sri Lanka, le dirigeant d'une organisation partenaire de Cordaid ajoute que «le gouvernement mène un combat contre les organisations de la société civile, combat qui s'inscrit dans le contexte mondial de la guerre contre le terrorisme. Toute organisation émettant des critiques sur la guerre contre le terrorisme est considérée comme suspecte et devient victime de contrôles abusifs, en raison de liens supposés avec des organisations terroristes 55 ».

50. L'Internal Security Act est un reliquat de l'occupation britannique. Il stipule que chaque individu peut être détenu 60 jours sans aucune forme d'accusation.

51 . Entretien réalisé en mai 2008 à Davao avec Latheefa Koya.

52 . Entretien réalisé en décembre 2008 à La Haye avec Ima Rociola, responsable au Salvador de l'organisation CEMUJER.

53. Entretien avec Babloo Loitongbam, La Haye, septembre 2008.

54. Voir par exemple Human Rights Watch, India. Getting Away With Murder. 50 years of the Armed Forces Special Powers Act, août 2008,

http://www.hrw.org/legacy/backgrounder/2008/india0808/

55 . Entretien réalisé à Davao en mai 2008 avec Sudarshana Gunawardana, avocat et dirigeant de Rights Now Collective for Democracy, une organisation des droits humains établie à 
Mener des activités humanitaires, de développement ou de paix dans des régions où les droits de l'homme ne sont pas respectés et où la distinction entre rebelles, terroristes et forces gouvernementales est ardue, constitue désormais une entreprise très délicate. Etre vu en train de discuter avec le leader d'une organisation dissidente constitue une condition suffisante pour être intimidé, accusé, arrêté ou inscrit sur une liste terroriste. Un exemple frappant concerne le directeur de SUDO, une organisation de développement soudanaise qui est partenaire de Cordaid : des agents de sécurité le suivent continuellement et son téléphone est sur écoute permanente ${ }^{56}$. La surveillance et les contrôles auxquels il est soumis se sont d'ailleurs accrus depuis que la Cour pénale internationale (CPI) a lancé un mandat d'arrêt contre le président Omar Al-Bashir. Soupçonné d'avoir fourni des informations à la CPI, il a du se présenter devant les tribunaux soudanais pour les accusations suivantes: atteinte à l'ordre public et collaboration avec les forces étrangères ${ }^{57}$. Depuis le lancement du mandat international contre Al-Bashir, les forces soudanaises de sécurité nationale accentuent aussi leurs pratiques d'intimidation à l'encontre des organisations locales travaillant dans l'humanitaire ou le développement, au nom de la lutte contre le terrorisme. Seize bureaux d'organisations ont été perquisitionnés et leur personnel a été molesté. Les abus inhérents aux mesures anti-terroristes prennent également la forme de tracasseries administratives, de fermetures des bureaux ou de confiscation de matériel.

Parallèlement les libertés des ONG sont aussi restreintes. En premier lieu, la liberté d'association. Depuis le 11 septembre, les conditions d'enregistrement des ONG deviennent drastiques. A titre d'exemple, la loi relative à l'enregistrement des ONG adoptée en 2006 en Ouganda limite la possibilité d'enregistrement pour les organisations œuvrant dans des domaines considérés comme «sensibles ». Au Soudan, le Voluntary Act de 2006 suit la même logique. Le gouvernement souhaite que les ONG se concentrent sur le domaine de l'humanitaire en se conformant aux priorités qu'il détermine. Il exige de pouvoir contrôler totalement les programmes de sécurité alimentaire. Son intervention dans ce domaine se traduit notamment par des lourdeurs bureaucratiques qui perturbent la distribution des vivres ${ }^{58}$. Les ONG deviennent de facto des agents d'exécution des gouvernements. Le gouvernement prend souvent l'initiative de fermer leurs bureaux, sans que cette décision ne repose sur aucun fondement légal. Par exemple, en 2007, plus de 40 associations de promotion de la paix et de défense des droits de l'homme ont été frappées par une telle mesure. L'organisation partenaire de Cordaid Gender Center for Research and Training basée à Khartoum n'a pu à nouveau ouvrir ses bureaux qu'après avoir signé une déclaration dans laquelle elle s'est enga-

Colombo au Sri Lanka.

56. Entretien réalisé par courrier électronique avec Ibrahim Mudawu, dirigeant de SUDO, juillet 2008.

57. Ibid.

58. Entretien avec Asha El-Karib, op. cit. 
gée à renoncer à la poursuite d'activités de nature politique ${ }^{59}$. Comme le souligne la dirigeante de cette organisation : «Au Soudan, le Gender Center est continuellement victime de sabotage de la part des services de sécurité. Le gouvernement s'octroie le droit de mener des investigations contre les ONG, de récupérer leurs activités ou de mettre un terme à leurs projets sans aucun recours judiciaire possible pour les ONG. En outre, les ONG sont censées se cantonner à fournir des services sociaux sous la supervision du gouvernement. Les ONG qui se lancent dans les domaines sensibles tels que les droits de l'homme et la démocratie sont perçues comme des ennemis de l'Etat $60 »$. La volonté gouvernementale de réguler les ONG n'est pas un phénomène nouveau. Néanmoins, la plupart des partenaires de Cordaid ayant participé aux ateliers qu'elle a organisés ont indiqué ressentir un resserrement très significatif des contrôles effectués par les services de sécurité depuis le 11 septembre.

En Ethiopie, les ONG recevant plus de $10 \%$ de fonds en provenance de l'étranger sont considérées comme des ONG internationales et toute immixtion dans les affaires internes du pays leur est interdite. Plus généralement, les gouvernements font pression sur ces organisations pour qu'elles s'alignent sur les politiques nationales sécuritaires poursuivant une logique de guerre contre le terrorisme, ce qui aboutit de facto à la « sécurisation » voire même à la «militarisation » de l'aide humanitaire et du développement. Un partenaire colombien souligne aussi que «les forces de sécurité sont de plus en plus visibles, certaines ONG ont leur comité de gestion infiltré par des militaires ou des membres des services de sécurité. Bref, le développement devient une question militaire $61 »$. Cette vision est partagée par une autre organisation partenaire de Cordaid dans ce pays qui explique combien la prédominance de logique sécuritaire aboutit à la «militarisation de la vie quotidienne 62 ».

On assiste aussi dans le même temps à une limitation de plus en plus grande de la circulation des membres des organisations. La plupart des ONG travaillant dans le secteur de la paix et de la réconciliation cherchent à rencontrer des opposants politiques afin de pouvoir susciter un dialogue susceptible de s'avérer propice au rétablissement d'une situation pacifiée. Or, voyager devient une entreprise de plus en plus difficile. Des missions de routine pour récolter des informations (fact finding mission) deviennent quasi impossibles. Les barrages érigés par les soldats et les forces de sécurité limitent les allers et venues ${ }^{63}$. Les partenaires de Cordaid soulignent pourtant l'importance pour les représentants des ONG d'avoir accès aux villages et aux populations qui

59. Ibid.

60. Ibid.

61. Entretien avec Francisco de Roux, op. cit.

62. Entretien réalisé à La Haye en décembre 2008 avec Beatriz Elena Quintero, membre de la Red Nacional de Mujeres, un réseau d'organisations féminines colombiennes créé en 1992 en Colombie.

63. Entretien avec Ruki Fernando, coordinateur du programme « Droits humains dans les situations de conflit » au sein de l'organisation LST (Law and Society Trust) au Sri Lanka. 
sont la première cible des combats et souvent victimes d'abus commis aussi bien par les groupes qualifiés de rebelles que par les forces gouvernementales ${ }^{64}$. Au Soudan, le HAC (Humanitarian Assistance Commitee, ou Comité d'assistance humanitaire) supervise toutes les demandes d'accès au Darfour. L'accès physique aux régions les plus sensibles demande aux ONG des efforts et une patience incommensurables. Y organiser un simple « atelier » devient une tâche insurmontable. Les noms, adresses, âges, et autres informations de tous les participants doivent généralement être attachés à la requête officielle qu'il est nécessaire d'adresser. Mentionner un nom douteux, suspect ou une activité inconnue accomplie par une personne peut à tout moment faire avorter ce type d'initiative. Au Sri Lanka, les restrictions de voyage engendrent un isolement des populations. Le voyage de Colombo à Jaffna dure normalement une heure en avion. En raison des normes de sécurité actuellement en vigueur destinées à contrecarrer les plans des terroristes, ce trajet s'effectue en dix heures. Outre les barrières physiques visant à limiter la liberté d'action du personnel des ONG et à tester leur patience, des "barrières financières »sont aussi érigées. Dans le cas du Sri Lanka, un billet d'avion pour se rendre de Colombo à Jaffna coûte plus cher qu'un billet international : "Ce n'est pas seulement une affaire de sécurité. C'est une stratégie militaire consciente dont l'objectif est d'empêcher les organisations sociales critiques à l'égard du gouvernement de travailler à Jaffna: l'accès humanitaire, l'accès physique aux personnes et aux communautés victimes des conflits et des calamités est remis en cause aussi bien par le terrorisme que par les mesures antiterroristes 65 ». De même, les voyages internationaux demandent une patience à toute épreuve. La responsable de l'organisation soudanaise Gender Centre for Research and Training in Khartoum a été arrêtée en 2006 à l'aéroport de New York pendant neuf heures et traitée comme une terroriste - subissant notamment des intimidations. L'ironie veut que des membres du gouvernement soudanais - un Etat dont les liens avec des organisations terroristes ont été prouvés - présents à bord du même avion ont pu circuler librement ${ }^{66}$.

A ces restrictions s'ajoutent celles concernant l'information. Le Regulation of Interception of Communication Bill 67 adopté en mai 2007 en Ouganda stipule que les services de sécurité peuvent intercepter toute infor-

Entretien réalisé à Davao (Philippines) à l'occasion d'une conférence organisée par Cordaid en mai 2008 dans cette ville sur l'impact des mesures antiterroristes sur les ONG.

64. "C'est important de rester en contact avec les communautés qui souffrent le plus des mesures anti-terroristes, et de leur faire part de ce qui se passe au delà des barricades et du front antiterroriste ", entretien avec Ruki Fernando, op. cit.

65. Ibid.

66. Entretien avec Asha El-Karib, op. cit.

67. Voir le dossier consacré par Amnesty International sur ce point. Selon Amnesty, cette loi a été adoptée en violation de certains traités internationaux, notamment le International Covenant on Civil and Political Rights (ICCPC) et le African Charter on Human and people's Rights (ACHPR),

http://www.amnesty.org/en/library/asset/AFR59/005/2008/en/10bf8327-7507-11dd-8e5e43ea85d15a69/afr590052008en.pdf 
mation sans autorisation judiciaire. De plus, la lutte anti-terroriste qui est menée dans ce pays alimente un processus de criminalisation des informations. En effet, en Ouganda, la loi antiterroriste de 2002 criminalise toute information susceptible de menacer la sécurité de l'Etat. Il en résulte que les ONG ont peur de publier des informations qui pourraient être interprétées comme relevant de cette catégorie ${ }^{68}$. Toujours en Ouganda, les téléphones portables du personnel d'une ONG partenaire de Cordaid, travaillant dans le domaine du genre et de la promotion des droits de la femme, ont été confisqués par les services de sécurité car leurs activités avaient été jugées suspectes ${ }^{69}$. Les services de sécurité exigent d'obtenir des informations sur toutes les activités organisées par les ONG aussi bien locales qu'internationales. Or, pour beaucoup de ces ONG, fournir des informations sur les bénéficiaires de leurs programmes équivaut à les exposer à des représailles ${ }^{70}$. En Colombie, la loi oblige les ONG à informer les services de renseignement et de sécurité de l'ensemble de leurs mouvements en «territoire rebelle » et interdit tout contact entre leaders communautaires. La seule exception concerne les dialogues «pastoraux » qui entrent dans le cadre de l'exercice de la fonction de prêtre. Les partenaires de Cordaid travaillant en Colombie dans le domaine de la paix et de la réconciliation et présents aux ateliers ont indiqué ressentir une accentuation de la pression exercée par le gouvernement. Selon eux, tout mouvement de personne, contact téléphonique ou email est étroitement surveillé ${ }^{71}$. Ce constat est partagé par la plupart des participants issus d'autres pays. Aux Philippines, chaque habitant de Sulu risque à tout moment d'être accusé d'avoir des liens avec les terroristes. Un climat de suspicion et de peur règne sur l'île. Les services de sécurité exigent de la population et des ONG locales qu'elles leur transmettent toute information jugée sensible ${ }^{72}$. Les services de sécurité surveillent en permanence les mouvements des membres des ONG locales et refuser de livrer des informations sur la population les expose à des représailles ${ }^{73}$. Au Sri Lanka, ce sont encore des docteurs ayant soigné des « rebelles » qui ont été contraints par les forces militaires à leur fournir des informations que ces personnes auraient pu leur divulguer ${ }^{74}$.

La guerre contre le terrorisme menée par de nombreux Etats dans les pays occidentaux ainsi que dans les pays en voie de développement réduit de façon

68. Entretien avec Livingstone Sewanyana réalisé à Kampala (Ouganda) en juillet 2008 durant l'atelier organisé dans cette ville par Cordaid. Livingstone Sewanyana est directeur exécutif de la Foundation for Human Rights Initiative, une organisation située à Kampala qui travaille autour de la thématique des droits humains.

69. Entretien avec Jessica Nkuuhe réalisé à Kampala en juillet 2008. Jessica Nkuuhe est une des personnalités du mouvement féministe africain. Actuellement consultante, elle a notamment travaillé comme directrice du Isis-WICCE (Women International Cross Cultural Exchange).

70. Entretien avec Ruki Fernando, op. cit.

71 . Entretien avec Francisco Le Roux, op. cit.

72. Entretien avec Octavio Dinampo, professeur d'histoire et de science politique à l'université de Mindanao. Il est aussi président d'un réseau d'activistes œuvrant pour la paix, le Mindanao Peoples Caucus. Entretien réalisé à Kampala en juillet 2008.

73. Ibid.

74. Echanges d'e-mails avec Ruki Fernando, 26 juin 2009. 
significative le champ d'action des organisations agissant aussi bien dans le monde de l'humanitaire que dans celui de la paix et du développement. Elle compromet les efforts déployés par ces organisations pour propager les valeurs démocratiques que promeuvent pourtant officiellement les Etats occidentaux. La question fondamentale qui se pose est de savoir si le vaste arsenal de mesures antiterroristes déployé est conciliable avec la liberté d'association qui constitue un des piliers de la vie démocratique. A travers cette question, c'est aussi celle du rôle que doivent jouer ces organisations qui se trouve posée. On ne peut en aucun cas les considérer comme des instruments au service soit des terroristes, soit de la logique sécuritaire de l'Etat. Un document produit en 2003 par le Comité d'aide au développement de l'OCDE intitulé «Une vision de coopération et de développement pour la prévention du terrorisme 75 » faisait état de la nécessité pour les Etats bailleurs de fonds de réduire le soutien au terrorisme en travaillant sur les causes profondes de l'émergence des conflits : l'exclusion sociale et l'injustice politique. La plupart des ONG sont d'accord avec cette assertion, même si des doutes sérieux sont émis quant à la possibilité de concilier ces deux logiques dans le contexte sécuritaire actuellement dominant.

75. OCDE, A development cooperation lens on terrorism prevention. Key entry points for Action, 2003, http://www.oecd.org/dataoecd/17/4/16085708.pdf 